\title{
PARABOLIC INITIAL BOUNDARY VALUE PROBLEMS IN NONSMOOTH CYLINDERS WITH DATA IN ANISOTROPIC BESOV SPACES
}

\author{
TÜNde JAKab ANd Marius Mitrea
}

\begin{abstract}
We prove sharp well-posedness results for the Poisson problem for the heat operator in arbitrary Lipschitz cylinders with Dirichlet and Neumann boundary conditions, when the boundary data are in parabolic Besov spaces.
\end{abstract}

\section{Introduction and statement of main results}

In the late 70's, E. Fabes and N. Rivière ( [FaRi]) have succeeded in solving the initial Dirichlet and Neumann problems for the heat operator in the cylindrical domain $\Omega \times(0, T)$ for a bounded $C^{1}$ domain $\Omega \subset \mathbb{R}^{n}$. They produced solutions to these problems satisfying the zero initial condition in the form of a caloric double layer potential in the Dirichlet case and a caloric single layer potential in the Neumann case. While the Neumann datum was taken from the Lebesgue space $L^{p}(\partial \Omega \times(0, T))$, $1<p<\infty$, the Dirichlet datum $g$ was assumed to be either from $L^{p}(\partial \Omega \times(0, T)), 1<$ $p<\infty$, or from the anisotropic Sobolev space $L_{1}^{p}(\partial \Omega \times(0, T))$, where the anisotropy is dictated by the heat operator.

When employed in the context of Dirichlet or Neumann problems, the method of layer potentials consists of reducing matters to inverting an operator of the form "identity $+K$ " acting on appropriate boundary function spaces. The functional analytic properties of $K$ are intimately linked to the smoothness assumptions made on $\partial \Omega$. When the domain $\partial \Omega \in C^{1}$, it has been observed that the operator in question is compact on the Lebesgue scale $L^{p}$, first in [FJR] for the case of the Laplace operator, then sortly thereafter in $[\mathrm{FaRi}]$ for the case of the heat operator. For domains whose boundary is only Lipschitz, $K$ may fail to be compact and this creates difficulties of a fundamental nature. In the case of the Laplacian these have been overcome by G. Verchota in [Ver] and B. Dahlberg and C. Kenig in [DaKe]. In 1990 R. Brown ([Bro]) was able to adapt this analysis to the case of the initial Dirichlet and Neumann boundary problems for the heat equation in Lipschitz cylinders, with zero initial data. The key ingredient in Brown's approach was establishing the invertibility of the appropriate caloric boundary layer potentials on Sobolev spaces with an integer amount of smoothness.

Received by the editors December 14, 2005.

2000 Mathematics Subject Classification. Primary: 35K05, 35B65; Secondary 31 B20.

Key words and phrases. Anisotropic Sobolev and Besov spaces, heat operator, Lipschitz cylinder, parabolic layer potentials, initial boundary value problems.

Authors supported in part by the NSF grants DMS - 0400639 and DMS-FRG - 0456306. 
In this note we take the next natural step and discuss an extension of Brown's results to the case when the data is allowed to have a fractional amount of smoothness (measured on Besov spaces with parabolic anisotropy). For the Laplace operator with a Dirichlet boundary condition, optimal results have been first proved by D. Jerison and C. Kenig in [JeKe] via harmonic measure techniques. Subsequently, the case of Neumann boundary conditions have been treated by E. Fabes, O. Mendez and M. Mitrea in $[\mathrm{FMM}]$ by employing the method of layer potentials. Here we initiate the study of parabolic problems and prove results akin those in [JeKe]-[FMM] for the heat operator $\Delta-\partial_{t}$ with Dirichlet and Neumann boundary conditions in arbitrary Lipschitz cylinders.

Our main results (extending a number of classical results in the literature - cf. [LiMa]) are as follows.

Theorem 1.1. For each bounded Lipschitz domain $\Omega$ in $\mathbb{R}^{n}$ there exists some small $\varepsilon>0$ with the following significance. Let $0<T<\infty$ and assume that the indices $1<p<\infty, 0<\alpha<1$ are such that the point with coordinates $\left(\alpha, \frac{1}{p}\right)$ belongs to the interior of the hexagonal region $O A B C D E$ depicted below:

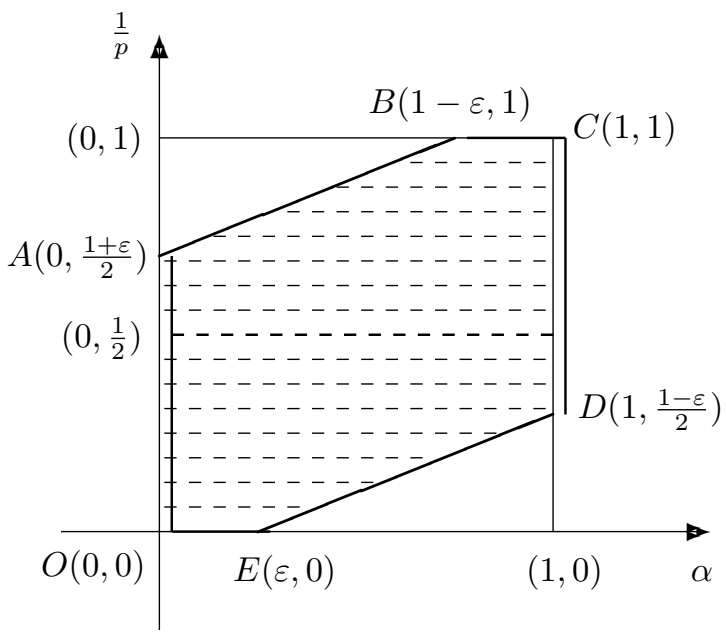

Figure 1.1

Then there exists a unique solution $u \in{ }_{0} B_{\alpha+\frac{1}{p}, p a r}^{p}(\Omega \times(0, T))$ to the problem

$$
(\mathrm{PD})\left\{\begin{array}{l}
\left(\partial_{t}-\Delta\right) u=f \in{ }_{0} B_{\alpha+\frac{1}{p}-2, p a r}^{p}(\Omega \times(0, T)), \\
\left.u\right|_{\partial \Omega \times(0, T)}=g \in{ }_{0} B_{\alpha, p a r}^{p}(\partial \Omega \times(0, T)) .
\end{array}\right.
$$

In addition, $u$ can be represented in terms of caloric layer potentials and

$$
\|u\|_{0} B_{\alpha+\frac{1}{p}, p a r}^{p}(\Omega \times(0, T)) \leq c\left(\|f\|_{0} B_{\alpha+\frac{1}{p}-2, p a r}^{p}(\Omega \times(0, T))+\|g\|_{0} B_{\alpha, p a r}^{p}(\partial \Omega \times(0, T))\right) .
$$

Corresponding to Neumann boundary conditions we have the following result.

Theorem 1.2. Given a bounded Lipschitz domain $\Omega$ in $\mathbb{R}^{n}$, there exists $\varepsilon>0$ such that if $0<T<\infty$ and $1<p<\infty, 0<\alpha<1$ are such that $\left(\alpha, 1-\frac{1}{p}\right) \in$ interior of 
$O A B C D E$, then there exists a unique solution $u \in{ }_{0} B_{1-\alpha+\frac{1}{p}, p a r}^{p}(\Omega \times(0, T))$ to the problem

$$
(\mathrm{PN})\left\{\begin{array}{l}
\left(\partial_{t}-\Delta\right) u=\left.f\right|_{\Omega \times(0, T)}, f \in{ }_{z} B_{-1-\alpha+\frac{1}{p}, p a r}^{p}(\Omega \times(0, T)), \\
\partial_{\nu}^{f} u=g \in{ }_{0} B_{-\alpha, p a r}^{p}(\partial \Omega \times(0, T)) .
\end{array}\right.
$$

Furthermore, there is an integral representation formula for the solution and

$$
\|u\|_{0} B_{1-\alpha+\frac{1}{p}, p a r}^{p}(\Omega \times(0, T)) \leq c\left(\|f\|_{z} B_{-1-\alpha+\frac{1}{p}, \text { par }}^{p}(\Omega \times(0, T))+\|g\|_{0} B_{-\alpha, p a r}^{p}(\partial \Omega \times(0, T))\right) .
$$

Above, $\partial_{\nu}^{f}$ can be thought of as a re-normalization of the normal derivative with respect to $f$. More detailed definitions are given in the next two sections, which also contain an outline of the proofs of Theorems 1.1-1.2.

\section{Smoothness function spaces with parabolic anisotropy}

One convenient way to introduce the parabolic Besov scale is via Littlewood-Paley theory. Specifically, fix $\varphi_{0} \in C^{\infty}\left(\mathbb{R}^{n} \times \mathbb{R}\right)$ such that $\varphi_{0}(x, t)=1$ if $\|(x, t)\|_{\text {par }} \leq 1$ and $\varphi_{0}(x, t)=0$ if $\|(x, t)\|_{\text {par }}>2$, where $\|(x, t)\|_{\text {par }}:=\left[|x|^{2}+|t|\right]^{1 / 2}$ for $x \in \mathbb{R}^{n}$, $t \in \mathbb{R}$ and, for each $j \in \mathbb{N}$, let $\varphi_{j}(x, t):=\varphi_{0}\left(2^{-j} x, 2^{-2 j} t\right)-\varphi_{0}\left(2^{-j+1} x, 2^{2(-j+1)} t\right)$. In particular, $\left\{\varphi_{j}\right\}_{j=0}^{\infty}$ is a partition of unity in $\mathbb{R}^{n} \times \mathbb{R}$. For $0<p, q \leq \infty, \alpha \in \mathbb{R}$, one then sets

$$
B_{\alpha, \text { par }}^{p, q}\left(\mathbb{R}^{n} \times \mathbb{R}\right):=\left\{f \in S^{\prime}\left(\mathbb{R}^{n} \times \mathbb{R}\right):\left(\sum_{j=0}^{\infty} 2^{j \alpha q}\left\|\mathcal{F}^{-1}\left(\varphi_{j} \mathcal{F} f\right)\right\|_{L^{p}\left(\mathbb{R}^{n} \times \mathbb{R}\right)}^{q}\right)^{1 / q}<\infty\right\},
$$

with the usual modification if $q=\infty$, where $\mathcal{F}$ and $\mathcal{F}^{-1}$ stand, respectively, for the Fourier transform and its inverse. Finally, set $B_{\alpha, p a r}^{p}\left(\mathbb{R}^{n} \times \mathbb{R}\right):=B_{\alpha, p a r}^{p, p}\left(\mathbb{R}^{n} \times \mathbb{R}\right)$.

Recall that a bounded domain $\Omega \subset \mathbb{R}^{n}$ is called Lipschitz provided its boundary can be locally described by means of (appropriately rotated and translated) graphs of real-valued Lipschitz functions defined in $\mathbb{R}^{n-1}$. Given a bounded Lipschitz domain $\Omega \subset \mathbb{R}^{n}$, the scale $B_{\alpha, p a r}^{p, q}(\partial \Omega \times \mathbb{R})$, with either $\frac{n+1}{n+2}<p, q \leq \infty,(n+1)\left(\frac{1}{p}-1\right)_{+}<$ $\alpha<1$, or $\frac{n+1}{n+2}<p, q<\infty,(n+1)\left(\frac{1}{p}-1\right)_{+}-1<\alpha<0$, where $(a)_{+}:=\max \{a, 0\}$, can be defined by transporting $B_{\alpha, p a r}^{p, q}\left(\mathbb{R}^{n-1} \times \mathbb{R}\right)$ to $\partial \Omega \times \mathbb{R}$ via localization (involving a smooth partition of unity), and pull-back.

Given the goals we have in mind, the next natural step is to introduce parabolic function spaces with built-in initial conditions. In concrete terms, we make the following.

Definition 2.1. Let $\Omega$ be a Lipschitz domain in $\mathbb{R}^{n}$ and $0<T<\infty$. For each $0<p, q \leq \infty$ and $\alpha \in \mathbb{R}$ we then set

$$
\begin{aligned}
& { }_{0} B_{\alpha, \text { par }}^{p, q}(\Omega \times(0, T)):=\left\{\left.F\right|_{\Omega \times(-\infty, T)}: F \in B_{\alpha, \text { par }}^{p, q}\left(\mathbb{R}^{n} \times \mathbb{R}\right), \text { supp } F \subseteq \mathbb{R}^{n} \times[0, \infty)\right\}, \\
& { }^{0} B_{\alpha, p a r}^{p, q}(\Omega \times(0, T)):=\left\{\left.F\right|_{\Omega \times(0, \infty)}: F \in B_{\alpha, p a r}^{p, q}\left(\mathbb{R}^{n} \times \mathbb{R}\right), \text { supp } F \subseteq \mathbb{R}^{n} \times(-\infty, T]\right\}, \\
& \text { and } \\
& { }_{z} B_{\alpha, p a r}^{p, q}(\Omega \times(0, T)):=\left\{\left.F\right|_{\mathbb{R}^{n} \times(-\infty, T)}: F \in B_{\alpha, p a r}^{p, q}\left(\mathbb{R}^{n} \times \mathbb{R}\right),\right. \\
& \text { supp } \left.F \subseteq(\bar{\Omega} \times[0, \infty)) \cup\left(\mathbb{R}^{n} \times[T, \infty)\right)\right\},
\end{aligned}
$$




$$
\begin{aligned}
{ }^{z} B_{\alpha, \text { par }}^{p, q}(\Omega \times(0, T)):=\left\{\left.F\right|_{\mathbb{R}^{n} \times(0, \infty)}:\right. & F \in B_{\alpha, \text { par }}^{p, q}\left(\mathbb{R}^{n} \times \mathbb{R}\right), \\
& \text { supp } \left.F \subseteq(\bar{\Omega} \times(-\infty, T]) \cup\left(\mathbb{R}^{n} \times(-\infty, 0]\right)\right\} .
\end{aligned}
$$

Also, on the lateral boundary of the cylinder $\Omega \times(0, T)$, for each $1<p, q<\infty$ and $\alpha \in(-1,1) \backslash\{0\}$, we set

${ }_{0} B_{\alpha, p a r}^{p, q}(\partial \Omega \times(0, T)):=\left\{\left.F\right|_{\partial \Omega \times(-\infty, T)}: F \in B_{\alpha, p a r}^{p, q}(\partial \Omega \times \mathbb{R})\right.$, supp $\left.F \subseteq \partial \Omega \times[0, \infty)\right\}$,

${ }^{0} B_{\alpha, p a r}^{p, q}(\partial \Omega \times(0, T)):=\left\{\left.F\right|_{\partial \Omega \times(0, \infty)}: F \in B_{\alpha, p a r}^{p, q}(\partial \Omega \times \mathbb{R})\right.$, supp $\left.F \subseteq \partial \Omega \times(-\infty, T]\right\}$.

These spaces are related via duality, as follows. If $1<p, q<\infty$ and $\frac{1}{p}+\frac{1}{p^{\prime}}=1$, $\frac{1}{q}+\frac{1}{q^{\prime}}=1$, then

$$
\begin{aligned}
& \left({ }^{0} B_{\alpha, \text { par }}^{p, q}(\Omega \times(0, T))\right)^{*}={ }_{z} B_{-\alpha, p a r}^{p^{\prime}, q^{\prime}}(\Omega \times(0, T)), \\
& \left({ }^{z} B_{\alpha, \text { par }}^{p, q}(\Omega \times(0, T))\right)^{*}={ }_{0} B_{-\alpha, p a r}^{p^{\prime}, q^{\prime}}(\Omega \times(0, T)),
\end{aligned}
$$

for $\alpha \in \mathbb{R} \backslash\{0\}$, and

$$
\left({ }_{0} B_{\alpha, p a r}^{p, q}(\partial \Omega \times(0, T))\right)^{*}={ }^{0} B_{-\alpha, p a r}^{p^{\prime}, q^{\prime}}(\partial \Omega \times(0, T)), \text { for } \alpha \in(-1,1) \backslash\{0\} .
$$

The spaces on $\Omega \times(0, T)$ are further related to those defined in $\partial \Omega \times(0, T)$ by means of traces. For example, given a bounded Lipschitz domain $\Omega$ in $\mathbb{R}^{n}$ and $0<T<\infty$, the trace mapping, originally defined as the restriction to the lateral boundary of functions continuous in $\bar{\Omega} \times[0, T]$, extends for each $\left(\alpha-\frac{1}{p}, \frac{1}{p}\right) \in$ interior of $O A B C D E$ to a bounded operator

$$
\operatorname{Tr}:{ }^{0} B_{\alpha, p a r}^{p}(\Omega \times(0, T)) \longrightarrow{ }^{0} B_{\alpha-\frac{1}{p}, p a r}^{p}(\partial \Omega \times(0, T))
$$

Similarly, $\operatorname{Tr}:{ }_{0} B_{\alpha, p a r}^{p}(\Omega \times(0, T)) \longrightarrow{ }_{0} B_{\alpha-\frac{1}{p}, p a r}^{p}(\partial \Omega \times(0, T))$.

Special care should be paid to the way the normal derivative is considered in (PN), as the function $u$ may not exhibit enough regularity in order to define $\partial_{\nu} u=\nu \cdot \operatorname{Tr}(\nabla u)$. To circumvent this problem, given a bounded Lipschitz domain $\Omega$ in $\mathbb{R}^{n}, 0<T<\infty$, and $1<p<\infty, 0<\alpha<1$, we let $\partial_{\nu}$ act on the space

$$
\begin{aligned}
\left\{(u, f) \in{ }_{0} B_{1-\alpha+1 / p, p a r}^{p}(\Omega \times(0, T)) \oplus{ }_{z} B_{-1-\alpha+1 / p, p a r}^{p}(\Omega \times(0, T)):\right. \\
\left.\left(\partial_{t}-\Delta\right) u=\left.f\right|_{\Omega \times(0, T)}\right\}
\end{aligned}
$$

by defining $\partial_{\nu}^{f} u \in{ }_{0} B_{-\alpha, p a r}^{p}(\partial \Omega \times(0, T))=\left({ }^{0} B_{\alpha, p a r}^{p^{\prime}}(\partial \Omega \times(0, T))\right)^{*}$ in the following fashion. If $\varphi \in{ }^{0} B_{\alpha, p a r}^{p^{\prime}}(\partial \Omega \times(0, T))$ and $\Phi \in{ }^{0} B_{\alpha+1 / p^{\prime}, p a r}^{p^{\prime}}(\Omega \times(0, T))$ is such that $\operatorname{Tr} \Phi=\varphi$, set

$$
\left\langle\partial_{\nu}^{f} u, \varphi\right\rangle:= \begin{cases}\langle\nabla u, \nabla \Phi\rangle-\langle f, \Phi\rangle+\left\langle\partial_{t} u, \Phi\right\rangle, & \text { if } \quad \alpha+\frac{1}{p}<1, \\ \langle\nabla u, \nabla \Phi\rangle-\langle f, \Phi\rangle-\left\langle u, \partial_{t} \Phi\right\rangle, & \text { if } \quad \alpha+\frac{1}{p}>1 .\end{cases}
$$

The case $\alpha+\frac{1}{p}=1$ is then covered by interpolation. For this program to work, at least when $\left(\alpha, \frac{1}{p^{\prime}}\right) \in$ interior of $O A B C D E$, one needs to know that the trace mapping in (3) has a continuous right inverse, and that the pairings in (4) are meaningful. The 
latter claim is a consequence of a Fubini-type property for parabolic Besov spaces which allows one to write

$$
B_{\alpha, p a r}^{p}(\Omega \times(0, T))=L^{p}\left(\Omega, B_{\alpha / 2}^{p}((0, T))\right) \cap L^{p}\left((0, T), B_{\alpha}^{p}(\Omega)\right),
$$

whenever $1 \leq p \leq \infty, \alpha>0$, with $B_{\alpha}^{p}$ denoting the standard (isotropic) Besov scale.

The parabolic Triebel-Lizorkin spaces $F_{\alpha, p a r}^{p, q}\left(\mathbb{R}^{n} \times \mathbb{R}\right)$ with $0<p<\infty, 0<q \leq \infty$ and $\alpha \in \mathbb{R}$, can be defined in a similar fashion; they satisfy properties which are analogous to those of parabolic Besov spaces. In addition, for each $\frac{n+2}{n+3}<p \leq 1$, the parabolic Hardy space $H_{\text {par }}^{1}\left(\mathbb{R}^{n} \times \mathbb{R}\right)$ can be identified with $F_{0, \text { par }}^{1,2}\left(\mathbb{R}^{n} \times \mathbb{R}\right)$, and we also set $H_{\text {par }}^{1, p}\left(\mathbb{R}^{n} \times \mathbb{R}\right):=F_{1, p a r}^{p, 2}\left(\mathbb{R}^{n} \times \mathbb{R}\right)$ for $\frac{n+2}{n+3}<p \leq 1$.

\section{Caloric layer potentials}

The strategy employed in the proof of our main results makes systematic use of layer potentials of parabolic type. More specifically, we let $\Pi$ denote the parabolic Newtonian potential, defined by formal convolution with the fundamental solution $E$ of the heat operator, i.e. for $(x, t) \in \mathbb{R}^{n} \times \mathbb{R}$,

$$
(\Pi f)(x, t):=\int_{\mathbb{R}^{n} \times \mathbb{R}} E(x-y, t-s) f(y, s) d y d s,
$$

where

$$
E(x, t):=\frac{1}{(4 \pi t)^{n / 2}} \exp \left\{\frac{-|x|^{2}}{4 t}\right\} \chi_{(0, \infty)}(t), \quad x \in \mathbb{R}^{n}, t \in \mathbb{R} .
$$

As a consequence of the Littlewood-Paley theory, this Newtonian potential is smoothing of order 2 on the Besov scale.

Next, introduce the single and double caloric layer potentials, respectively, as

$$
\begin{aligned}
\mathcal{D} f(x, t) & :=\int_{0}^{t} \int_{\partial \Omega} \partial_{\nu_{y}}[E(x-y, t-s)] f(y, s) d \sigma_{y} d s, \quad(x, t) \in \Omega \times(0, T), \\
\mathcal{S} f(x, t) & :=\int_{0}^{t} \int_{\partial \Omega} E(x-y, t-s) f(y, s) d \sigma_{y} d s, \quad(x, t) \in \Omega \times(0, T),
\end{aligned}
$$

where $d \sigma$ and $\nu$ denote, respectively, the surface measure and the unit normal on $\partial \Omega$. Consider also

$$
K f(x, t):=\lim _{\varepsilon \rightarrow 0^{+}} \int_{0}^{t-\varepsilon} \int_{\partial \Omega} \partial_{\nu_{y}}[E(x-y, t-s)] f(y, s) d \sigma_{y} d s, \quad(x, t) \in \partial \Omega \times(0, T),
$$

and, if $R f(x, t):=f(x, T-t)$ is a reflection in time, set $K^{\prime}:=R \circ K^{*} \circ R$, where $K^{*}$ is the formal adjoint of $K$.

The idea is then to look for solutions of the initial boundary problems (PD), (PN) in the form

$$
u=\Pi f+\mathcal{D}\left[\left(\frac{1}{2} I+K\right)^{-1}(g-\operatorname{Tr}(\Pi f))\right]
$$

and, respectively,

$$
u=\Pi f+\mathcal{S}\left[\left(-\frac{1}{2} I+K^{\prime}\right)^{-1}\left(g-\partial_{\nu}^{f} \Pi f\right)\right] .
$$

The incisive result, pertaining to the mapping properties of these operators, reads as follows. 
Theorem 3.1. Let $\Omega$ be a bounded Lipschitz domain in $\mathbb{R}^{n}, 0<T<\infty$ and $0<$ $\alpha<1,1<p<\infty$ such that $\left(\alpha, \frac{1}{p}\right) \in$ interior of $O A B C D E$. Then

$$
\begin{array}{ll}
\mathcal{D} & : \quad{ }_{0} B_{\alpha, p a r}^{p}(\partial \Omega \times(0, T)) \longrightarrow{ }_{0} B_{\alpha+1 / p, p a r}^{p}(\Omega \times(0, T)), \\
\mathcal{S} & : \quad{ }_{0} B_{-\alpha, p a r}^{p}(\partial \Omega \times(0, T)) \longrightarrow{ }_{0} B_{1-\alpha+1 / p, p a r}^{p}(\Omega \times(0, T)),
\end{array}
$$

are bounded. Also, with $1 / p+1 / p^{\prime}=1$, the operators

$$
\begin{array}{rll}
\frac{1}{2} I+K & : & { }_{0} B_{\alpha, p a r}^{p}(\partial \Omega \times(0, T)) \stackrel{\sim}{\longrightarrow}{ }_{0} B_{\alpha, p a r}^{p}(\partial \Omega \times(0, T)), \\
-\frac{1}{2} I+K^{\prime} & : & { }_{0} B_{-\alpha, p a r}^{p^{\prime}}(\partial \Omega \times(0, T)) \stackrel{\sim}{\longrightarrow}{ }_{0} B_{-\alpha, p a r}^{p^{\prime}}(\partial \Omega \times(0, T)),
\end{array}
$$

are invertible, and

$$
\operatorname{Tr} \circ \mathcal{D}=\frac{1}{2} I+K, \quad \partial_{\nu}^{0} \mathcal{S}=-\frac{1}{2} I+K^{\prime} .
$$

The key step in proving the aforementioned mapping properties for the caloric double layer is establishing two basic estimates. Concretely, if $\delta(x, t):=\operatorname{dist}(x, \partial \Omega)$, $0<\alpha<1, \theta \geq 0, k \in \mathbb{N} \cup\{0\}$ then, for $N:=k+2 \theta-\alpha-1>-1$,

$$
\begin{aligned}
\delta^{N} D_{t}^{\theta} \nabla_{x}^{k} \mathcal{D} & : \quad B_{\alpha, \text { par }}^{1}(\partial \Omega \times \mathbb{R}) \longrightarrow L^{1}(\Omega \times \mathbb{R}), \\
\delta^{N+1} D_{t}^{\theta} \nabla_{x}^{k} \mathcal{D} & : \quad B_{\alpha, \text { par }}^{\infty}(\partial \Omega \times \mathbb{R}) \longrightarrow L^{\infty}(\Omega \times \mathbb{R}),
\end{aligned}
$$

where $D_{t}^{\theta}$ stands for the fractional order operator of order $\theta$ in the time variable. Once this is accomplished, E. Stein's interpolation theorem for analytic families of operators yields that

$$
\delta^{N+1-\frac{1}{p}} D_{t}^{\theta} \nabla_{x}^{k} \mathcal{D}: B_{\alpha, p a r}^{p}(\partial \Omega \times \mathbb{R}) \longrightarrow L^{p}(\Omega \times \mathbb{R}) \text { whenever } 1 \leq p \leq \infty .
$$

This latter property can then be re-interpreted in terms of membership to a parabolic Besov space in the Lipschitz cylinder $\Omega \times \mathbb{R}$, thus yielding the desired conclusion in regard to $\mathcal{D}$. Furthermore, a similar approach works in the case of the caloric single layer.

As regards the invertibility properties of the boundary caloric layer potentials, the departure point is to recall that R. Brown has proved that $\pm \frac{1}{2} I+K$ are isomorphisms of ${ }_{0} F_{0, p a r}^{p, 2}(\partial \Omega \times(0, T))$ for $2-\varepsilon<p<\infty$ and of ${ }_{0} F_{1, p a r}^{p, 2}(\partial \Omega \times(0, T))$ for $1 \leq p<2+\varepsilon$. Given that being an isomorphism is a stable property on a complex interpolation scale, the latter result can be perturbed yielding the existence of some $\eta=\eta(\partial \Omega)>0$ such that the operators

$$
\pm \frac{1}{2} I+K \text { are isomorphisms of }{ }_{0} H_{\text {par }}^{1, p}(\partial \Omega \times(0, T)) \text { whenever } 1-\eta<p \leq 1 .
$$

Since being an invertible operator is also preserved by passing from ${ }_{0} H_{p a r}^{1, p}(\partial \Omega \times(0, T))$ to the smallest Banach space which contains it, that is, ${ }_{0} B_{1-\alpha, p a r}^{1}(\partial \Omega \times(0, T))$ with $\alpha=(n+1)\left(\frac{1}{p}-1\right)$, this finally yields that

(3) $\quad \pm \frac{1}{2} I+K$ are isomorphisms on ${ }_{0} B_{1-\alpha, p a r}^{1}(\partial \Omega \times(0, T))$ for $\alpha>0$ small.

This takes care of the invertibility of $\pm \frac{1}{2} I+K$ on the horizontal segment $B C$ in the diagram in Figure 1.1, and we are left with discussing the case of the horizontal segment $O E$.

To this end, we start from the fact that the operators $\pm \frac{1}{2} I+K^{\prime}$ are bounded on ${ }_{0} F_{0, p a r}^{p, 2}(\partial \Omega \times(0, T))$ for $\frac{n+1}{n+2}<p<\infty$ and are isomorphisms when $p=1$ (cf. [Bro] 
for the latter claim). Perturbing this result yields that $\pm \frac{1}{2} I+K^{\prime}$ are invertible on ${ }_{0} H_{\text {par }}^{p}(\partial \Omega \times(0, T))$ for $p<1$ sufficiently close to 1 . Dualizing this and using the fact that, for $\frac{n+1}{n+2}<p<1$ and $\alpha=(n+1)\left(\frac{1}{p}-1\right)$,

$$
\left({ }_{0} H_{\text {par }}^{p}(\partial \Omega \times(0, T))\right)^{*}={ }^{0} B_{\alpha, p a r}^{\infty}(\partial \Omega \times(0, T)),
$$

we obtain that there exists $\eta=\eta(\partial \Omega)>0$ such that

$$
\pm \frac{1}{2} I+K \text { are invertible on }{ }_{0} B_{\alpha, p a r}^{\infty}(\partial \Omega \times(0, T))
$$

$$
\text { for } \alpha=(n+1)\left(\frac{1}{p}-1\right) \text { and } 1-\eta<p<1 \text {. }
$$

This takes care of the invertibility of $\pm \frac{1}{2} I+K$ on the horizontal segment $O E$ in the diagram in Figure 1.1. At this point, the interior of the entire region $O A B C D E$ is covered by repeated applications of the complex and real methods of interpolation.

In closing, we would like to point out that we may take $\varepsilon=1$ in the diagram in Figure 1.1 if $\partial \Omega \in C^{1}$ (compare with [FaRi]).

\section{References}

[Bro] R. M. Brown, The initial-Neumann problem for the heat equation in Lipschitz cylinders, Trans. Amer. Math. Soc. 320 (1990), no. 1, 1-52.

[DaKe] B.E. J. Dahlberg and C.E.Kenig, Hardy spaces and the Neumann problem in $L^{p}$ for Laplace's equation in Lipschitz domains, Ann. of Math. (2) 125 (1987), no. 3, 437-465.

[FJR] E. Fabes, M. Jodeit, Jr. and N. M. Rivière, Potential techniques for boundary value problems on $C^{1}$-domains, Acta Math. 141 (1978), no. 3-4, 165-186.

[FMM] E. Fabes, O. Mendez and M. Mitrea, Boundary layers on Sobolev-Besov spaces and Poisson's equation for the Laplacian in Lipschitz domains, J. Funct. Anal. 159 (1998) 323-368.

[FaRi] E. B. Fabes and N.M. Rivière, Dirichlet and Neumann problems for the heat equation in $C^{1}$-cylinders, pp. 179-196 in "Harmonic Analysis in Euclidean Spaces", Proc. Sympos. Pure Math., Vol. XXXV, Amer. Math. Soc., Providence, R.I., 1979.

[JeKe] D. Jerison and C. E. Kenig, The inhomogeneous Dirichlet problem in Lipschitz domains, J. Funct. Anal. 130 (1995) 161-219.

[KaMi] N. Kalton and M. Mitrea, Stability results on interpolation scales of quasi-Banach spaces and applications, Trans. Amer. Math. Soc. 350 (1998), no. 10, 3903-3922.

[LiMa] J.L. Lions and E. Magenes, Non-homogeneous boundary value problems and applications, Vol. I, II, III. Springer-Verlag, 1972.

[Ver] G. Verchota, Layer potentials and boundary value problems for Laplace's equation in Lipschitz domains, Ph.D. Thesis, University of Minnesota, 1982.

Department of Mathematics, University of Missouri, Columbia, Missouri 65211

E-mail address: jakabt@gmail.com

E-mail address: marius@math.missouri.edu 